\title{
二聚锰络合物催化的选择性内炔氢芳基化反应
}

\author{
刘旭芳刘强*
}

(清华大学化学系 基础分子科学中心 北京 100084)

\section{A Highly Efficient Dimeric Manganese-Catalyzed Selective Hydroarylation of Internal Alkynes}

\author{
Liu, Xufang Liu, Qiang*
}

(Center of Basic Molecular Science, Department of Chemistry, Tsinghua University, Beijing 100084)

过渡金属催化的内炔氢官能团化反应是合成多取 代烯烃分子的一种重要方法, 实现化学选择性、区域选 择性以及立体选择性的协同控制是其中的难点所在 ${ }^{[1]}$. 芳基硼酸试剂因其商业可得以及易于操作等特性, 已经 被广泛应用于炔烃氢芳基化反应(Scheme 1a). 目前, 通 过采用电子效应或者导向基团策略, 利用钯 ${ }^{[2]}$ 、铑 ${ }^{[3]}$ 、 铜 ${ }^{[4]}$ 、镍 ${ }^{[5]}$ 等催化剂均可以实现该反应转化. 然而, 这些 金属的高反应活性在一定程度上降低了反应的化学选 择性. 此外, 导向基团的引入以及移除通常需要多步的 分离纯化过程, 从而导致此类反应效率较低, 原子经济 性较差.

$\gamma$-芳基取代的三级烯丙基醇化合物是一类重要的有 机合成砌块, 并且普遍存在于众多生物活性分子中. 然 而, 对于此类化合物的高效合成方法却鲜有报道. 因此, 南京大学化学化工学院谢劲课题组设想通过大位阻的 三级炔丙基醇与芳基硼酸的 $\gamma$-选择性氢芳基化反应实 现目标分子的高效直接构建.

锰元素是地壳中储量第三的丰产过渡金属元素, 由 于其毒性低、官能团兼容性好, 金属锰配合物已被广泛 用于催化芳烃导向碳氢键活化一炔烃氢芳基化反应 ${ }^{[6]}$. 而利用芳基硼酸试剂替代含有导向基团的芳烃底物可 以进一步拓展芳基化产物类型. 此前, 谢劲团队报道了 锰催化的芳基硼酸与炔丙基醇的 $\beta$-选择性氢芳基化反

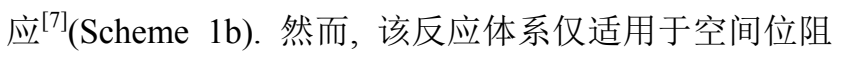
较小的炔丙基醇底物, 并且反应的区域选择性极大地受 限于取代基团的电子效应. 最近, 谢劲课题组提出了一 类 “双重控制” 策略，结合立体位阻效应以及羟基导向 作用, 成功实现了锰催化的炔丙基醇的 $\gamma$-选择性氢芳基
化反应 ${ }^{[8]}$.

(a) The current status of transition metal-catalyzed hydroarylation

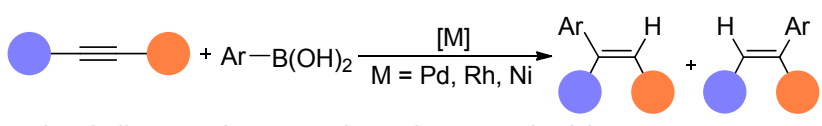

major challenges: chemo-, regio- and stereo-selectivity

(b) Previous work: $\beta$-selective hydroarylation of propargyl alcohols

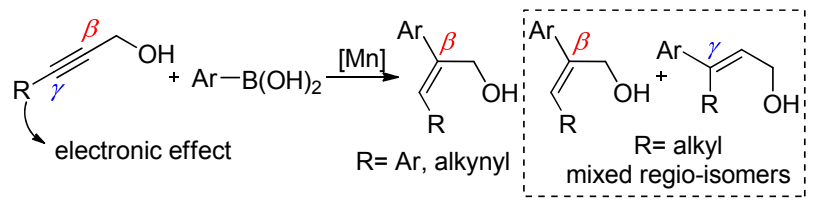

(c) This work: $\gamma$-selective hydroarylation of bulky propargyl alcohols
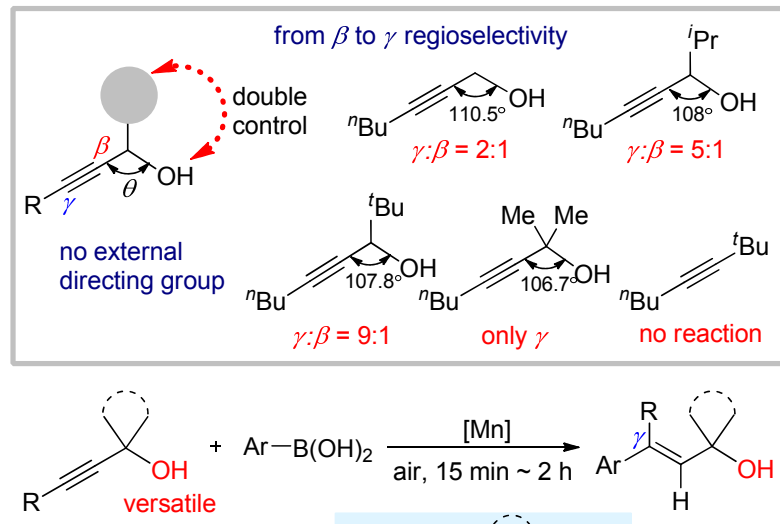

$\mathrm{R}=$ aryl, alkyl, alkenyl,alknyl

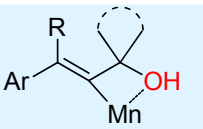

no $\beta$-oxygen elimination

图式 1 过渡金属催化的内炔氢芳基化反应

Scheme 1 Transition metal-catalyzed hydroarylation of internal alkynes

* Corresponding author. E-mail: qiang_liu@mail.tsinghua.edu.cn. Published online August 3. 2020. 
如 Scheme 1c 所示, $\theta$ 夹角越小, $\gamma$ 选择性越高, 当底 物分子中引入三级醇结构时，仅获得 $\gamma$-芳基化产物，而 不含有羟基的大位阻炔烃底物则完全没有反应活性, 由 此可以说明 “位阻因素” 和 “羟基配位” 对于反应的高 $\gamma$ 选择性控制缺一不可.

使用二聚 $\mathrm{Mn}_{2}(\mathrm{CO})_{8} \mathrm{Br}_{2}(5 \mathrm{~mol} \%)$ 作为催化剂前体, 加入 2.5 equiv. $\mathrm{KHCO}_{3}$ 以及 10 equiv. $\mathrm{H}_{2} \mathrm{O}$, 在空气条件 下可顺利实现一系列 $\gamma$-芳基-三级烯丙基醇类化合物的 高化学选择性、区域选择性以及立体选择性合成 (Scheme 2). 该催化反应体系对于各类取代内炔及芳基
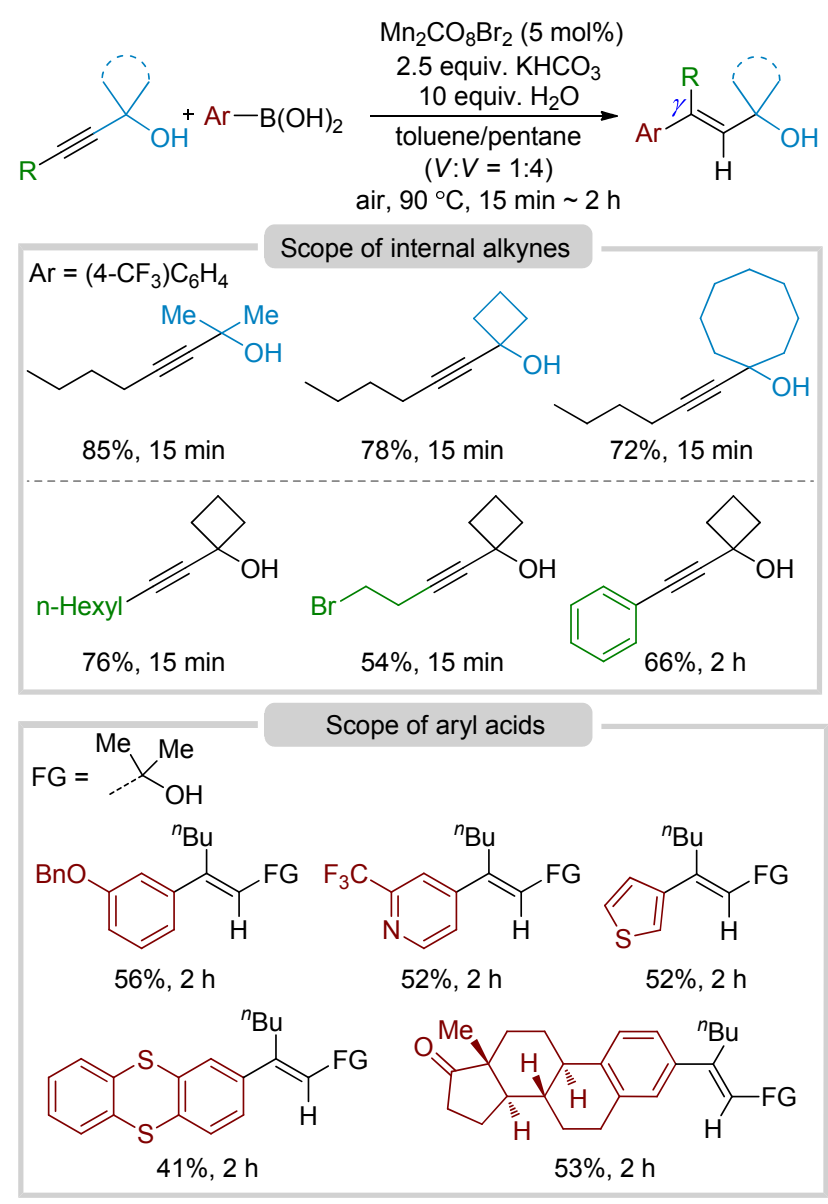

图式 2 内炔及芳基硼酸底物拓展

Scheme 2 Substrate scope of internal alkynes and aryl acids 嗍酸均体现出广泛的底物适用性, 共计合成超过 90 个
氢芳基化目标产物分子，产率最高可达 95\%. 值得一提 的是，反应的选择性控制并不依赖于底物中取代基团的 “电子效应”，脂肪族以及芳香族炔烃均可兼容.

此外，作者也开展了相关的合成应用研究，利用锰 催化 $\gamma$-氢芳基化反应策略, 通过 4 步反应即可实现降血 脂活性药物 Bervastatin 的高效合成(Scheme 3).

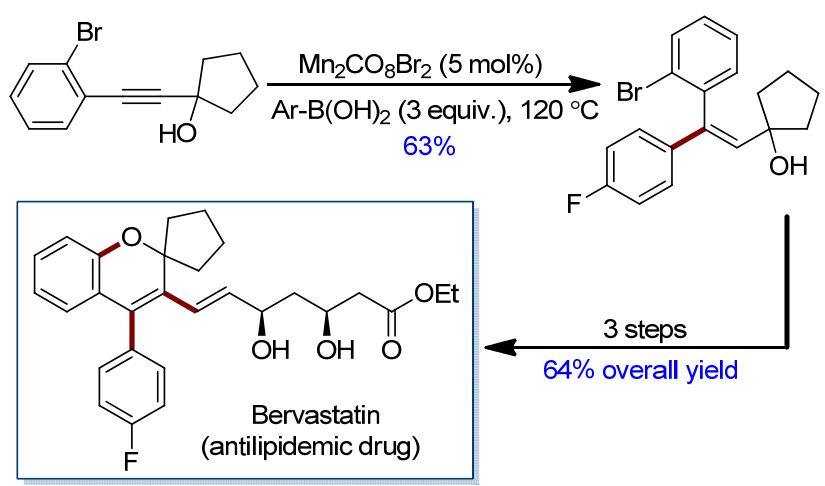

图式 3 药物分子合成

Scheme 3 Synthesis of drug molecule bervastatin

综上所述, 谢劲课题组发展了首例锰催化的大位阻 炔丙基醇的 $\gamma$-选择性氢芳基化反应，为一系列 $\gamma$-芳基三级烯丙基醇类化合物的高效合成提供了新的方法和 思路. 该策略的发展对于取代烯烃合成以及药物分子的 研发具有重要启发意义与应用价值.

\section{References}

[1] (a) Robbins, D. W.; Hartwig, J. F. Science 2011, 333, 1423.

[2] (a) Oh, C. H.; Jung, H. H.; Kim, K. S.; Kim, N. Angew. Chem., Int. Ed. 2003, 42,805 .

(b) Liu, Z.; Derosa, J.; Engle, K. M. J. Am. Chem. Soc. 2016, 138, 13076.

[3] Fagnou, K.; Lautens, M. Chem. Rev. 2003, 103, 169.

[4] Yamamoto, Y.; Kirai, N.; Harada, Y. Chem. Commun. 2008, 2010.

[5] Hari Babu, M.; Ranjith Kumar, G.; Kant, R.; Sridhar Reddy, M. Chem. Commun. 2017, 53, 3894.

[6] Hammarback, L. A.; Clark, I. P.; Sazanovich, I. V.; Towrie, M.; Robinson, A.; Clarke, F.; Meyer, S.; Fairlamb, I. J. S.; Lynam, J. M. Nat. Catal. 2018, 1, 830.

[7] Yan, Z.; Yuan, X.-A.; Zhao, Y.; Zhu, C.; Xie, J. Angew. Chem., Int Ed. 2018, 57, 12906.

[8] Pang, Y.; Liu, G.; Huang, C.; Yuan, X. A.; Li, W.; Xie, J. Angew. Chem., Int. Ed. 2020, 59, 12789. 